\title{
Geranylgeraniol Induces PPAR $\gamma$ Expression and Enhances the Biological Effects of a PPAR $\gamma$ Agonist in Adipocyte Lineage Cells
}

\author{
TAKUMA MATSUBARA ${ }^{1}$, NANA TAKAKURA ${ }^{1}$, MARIKO URATA $^{1}$, YUYA MURAMATSU $^{1}$, MAKOTO TSUBOI $^{1}$, \\ KAZUMA YASUDA ${ }^{1}$, WILLIAM N. ADDISON ${ }^{2}$, MIN ZHANG $^{3}$, KOU MATSUO $^{3}$, CHIHIRO NAKATOMI $^{1}$, \\ YUKIYO SHIGEYAMA-TADA ${ }^{4}$, TAKESHI KANEUJI ${ }^{5}$, ATSUKO NAKAMICHI ${ }^{6}$ and SHOICHIRO KOKABU ${ }^{1}$ \\ Divisions of ${ }^{1}$ Molecular Signaling and Biochemistry, and ${ }^{3}$ Oral Pathology, Department of Health Promotion, \\ ${ }^{4}$ Division of Dental Anesthesiology, Department of Control of Physical Functions, and \\ ${ }^{6}$ Department of Oral Functional Management, School of Oral Health Sciences, \\ Kyushu Dental University, Kitakyushu, Japan; \\ ${ }^{2}$ Research Unit, Shriners Hospitals for Children-Canada, Department of Human Genetics, \\ McGill University, Montreal, QC, Canada; \\ ${ }^{5}$ Division of Oral and Maxillofacial Surgery, Department of Medicine of Sensory and Motor Organs, \\ Faculty of Medicine, University of Miyazaki, Miyazaki, Japan
}

\begin{abstract}
Background: The global incidence of diabetes mellitus (DM) has risen precipitously, even in middle- and low-income countries. Peroxisome proliferator-activated receptor $\gamma(P P A R \gamma)$ plays an important role in the control of cellular glucose metabolism. Activation of PPAR $\gamma$ beneficially results in increased insulin sensitivity. However, the expression of PPAR $\gamma$ is reduced by obesity and several nutritional factors. Here we examined the effect of geranylgeraniol (GGOH), a bioactive compound found naturally in fruits, vegetables, and grains, on the expression and activation of PPAR $\gamma$. Materials and Methods: C3H1OT1/2 mouse embryonic fibroblasts and 3T3-L1 preadipocytes were used as in vitro models of adipocyte differentiation and function. Quantitative reversetranscriptase polymerase chain reaction, western blotting, Oil Red $O$ staining, and luciferase assay were performed to respectively assess $m R N A$ expression, protein levels, lipid droplet formation and transcriptional activity. Results:
\end{abstract}

This article is freely accessible online.

Correspondence to: Shoichiro Kokabu, D.D.S, Ph.D., Division of Molecular Signaling and Biochemistry, Department of Health Promotion, Kyushu Dental University, 2-6-1 Kitakyushu, Fukuoka 803-8580, Japan. Tel: +81 932851131, Fax: +81 932856000, e-mail: r14kokabu@fa.kyu-dent.ac.jp

Key Words: Diabetes, adipogenesis, statin, antidiabetic drug, geranylgeranylation.
GGOH increased the expression of PPAR $\gamma$ in adipocyte lineage cells. GGOH also enhanced adipogenesis induced by rosiglitazone, a thiazolidinedione class PPAR $\gamma$ agonist. Conclusion: GGOH induces PPAR $\gamma$ expression and enhances the biological effects of a PPAR $\gamma$ agonist in adipocyte lineage cells.

Diabetes mellitus (DM), one of the major chronic metabolic diseases, occurs either when pancreatic $\beta$-cells do not produce enough insulin or when the body does not respond efficiently to insulin. Insulin is a key regulator of blood sugar level. DM is divided into type 1 and type 2 . Type 1 results from defective insulin production from pancreatic $\beta$ cells. Type 2 arises from the body's ineffective usage of insulin. Type 2 is the most common form of DM worldwide. $\mathrm{DM}$ is a major cause of kidney failure, blindness, stroke, heart attack, and inferior limb amputation. In 2014, DM occurred in $8.5 \%$ of adults over the age of 18 years. In 2012, DM was the direct cause of 1.5 million deaths and conditions involving high blood sugar levels caused another 2.2 million deaths (1). Therefore, DM is worldwide problem in need of immediate attention.

Peroxisome proliferator-activated receptor $\gamma(\operatorname{PPAR} \gamma)$ is a nuclear hormone receptor, which plays an important role in controlling the metabolism of cellular glucose. PPAR $\gamma$ is mainly expressed in adipocytes and immune system cells (2, 3). Two PPAR $\gamma$ isoforms arising from the usage of alternate promoters and RNA splicing have been identified in humans and mice (2). PPAR $\gamma 2$ differs from PPAR $\gamma 1$ due to an additional 28 amino acids at the amino terminus. Activation 
of PPAR $\gamma$ increases insulin sensitivity thus resulting in a lower serum glucose concentration (4). In human adipocytes, insulin can in turn increase the expression of both PPAR $\gamma 1$ and PPAR $\gamma 2$. However, obesity and nutritional factors appear to reduce the expression only of PPAR $\gamma 2$ (5). PPAR $\gamma$ agonists are, therefore, being explored as potential antidiabetic drugs. Indeed, thiazolidinediones, such as rosiglitazone, are synthetic PPAR $\gamma$ agonists that are clinically used to maintain glucose homeostasis and enhance insulin sensitivity (6). PPAR $\gamma$ receptors can also be stimulated by the binding of micromolar concentrations of various kinds of lipophilic ligands, such as polyunsaturated fatty acids and eicosanoid derivatives, to the PPAR $\gamma$ receptor (7).

Geranylgeraniol $(\mathrm{GGOH})$ is a $\mathrm{C} 20$ isoprenoid found in fruits, vegetables, and grains, including rice. As a food substance, GGOH is categorized as 'Generally Recognized as Safe' (8). GGOH is an intermediate product in the mevalonate pathway and acts as a precursor to geranylgeranylpyrophosphate (GGPP). In the cell, GGOH is thought to be subsequently converted into the pyrophosphate moiety, GGPP, by two successive monophosphorylation events $(9,10)$. GGPP-induced geranylgeranylation is needed for the membrane anchoring of intracellular proteins, especially the small GTP-binding proteins RHO, RAC, RAS and RAP, which are involved in several signaling pathways (11). In cell-based studies, GGPP treatment led to increased expression of PPAR $\gamma$ (12). Statins, such as simvastatin, are a class of lipid-lowering compounds which inhibit hydroxymethylglutaryl-CoA (HMG-CoA) reductase. HMGCoA reductase is utilized in the initial step in the biosynthetic pathway of isoprenoid, as well as the ratecontrolling step of cholesterol biosynthesis (13). Simvastatin reduces the expression of PPAR $\gamma$ and also inhibits adipogenesis (14).

\section{Materials and Methods}

Cell culture. C3H10T1/2 mouse embryonic fibroblasts and 3T3-L1 pre-adipocytes were purchased from the American Type Culture Collection (Manassas, VA, USA). Cells were cultured in Dulbecco's modified Eagle's medium containing $10 \%$ fetal bovine serum (15, 16). Cells were cultured in the presence of different concentrations $(0,5,10,50,100 \mu \mathrm{M})$ of GGOH (LKT Laboratories, Inc., St. Paul, MN, USA), $10 \mu \mathrm{M}$ rosiglitazone (Wako, Osaka, Japan) and 100 or $200 \mu$ M Simvastatin Natrium (Wako).

Reverse transcription and quantitative polymerase chain reaction $(q P C R)$ analysis. Total RNA was isolated from cells using Trizol Reagents (Thermo Fisher Scientific, Waltham, MA, USA) and then reverse-transcribed into cDNA using ReverTra Ace (Toyobo, Osaka, Japan). The cDNA was amplified by PCR using specific primers for murine Pparg2 (forward, tgctgttatgggtgaaactctg; reverse, ctgtgtcaa ccatggtaatttctt), fatty acid binding protein 4 (Fabp4) (forward, ggatggaaagtcgaccacaa; reverse, tggaagtcacgcctttcata); CCAATenhancer-binding protein $\alpha$ (Cebpa) (forward, aaacaacgcaa cgtggaga; reverse, gcggtcattgtcactggtc); adiponectin, $\mathrm{C} 1 \mathrm{Q}$ and collagen domain-containing (Adipoq) (forward, ggagagaaaggaga tgcaggt; reverse, ctttcctgccaggggttc); and $\beta$-actin (Actb) (forward, aaggccaaccgtgaaaagat; reverse, gtggtacgaccagaggcatac). SYBR green-based qPCR was performed using PowerUp SYZBR Green Master Mix (ThermoFisher Scientific) with QuantStudio 3 system (Thermo Fisher Scientific). Expression values were normalized to those for Actb using the $2-\Delta \Delta \mathrm{Ct}$ method (17).

Transfection and luciferase assay. Cells were transfected with FABP4 (aP2)-luc plasmid (18) and phRL-SV40 (Promega, Madison, WI, USA) with or without FLAG-PPARG2 (18), using Lipofectamine 2000 (Thermo Fisher Scientific) according to the manufacturer's instructions. Luciferase assay was performed using the Dual-Glo Luciferase Assay System (Promega) as described previously (19).

Western blot analysis. The following antibodies were used for western blot analysis: anti-PPAR $\gamma$ (rabbit monoclonal antibody; Cell Signaling, Beverly, MA, USA), anti-FABP4 (rabbit monoclonal antibody; Cell Signaling), and anti- $\beta$-actin (mouse monoclonal antibody; Sigma Aldrich Chemicals, St. Louis, MO, USA). The target proteins were detected using an anti-mouse or anti-rabbit IgG antibody conjugated with horseradish peroxidase (Cell Signaling) and visualized by ImmunoStar LD (Wako).

Oil red $O$ staining. C3H10T1/2s cell and 3T3-L1 cells were treated with $10 \mu \mathrm{M}$ rosiglitazone (Wako) to induced adipocyte differentiation. On day 7 , cells were rinsed twice with phosphatebuffered saline, fixed in $4 \%$ paraformaldehyde and stained with oil red O (Sigma) for 10 minutes at room temperature (18).

Statistical analysis. Comparisons were made using Wilcoxon's signed-rank test and unpaired ANOVA with Tukey-Kramer post-hoc test. The results are shown as the mean \pm S.D. The statistical significance was accepted at values of $p<0.05$.

\section{Results}

GGOH induces the expression of PPAR $\gamma$ and enhances the biological effect of PPAR $\gamma$ agonist. Firstly, it was examined whether GGOH affects the expression of PPAR $\gamma$ in C3H10T1/2 and 3T3-L1 cells. C3H10T1/2 mouse embryonic fibroblasts and 3T3-L1 pre-adipocytes express PPAR $\gamma 2$ and have a capacity for adipogenesis in response to PPAR $\gamma$ agonists (20). Treatment of C3H10T1/2 cells with GGOH significantly increased messenger RNA (mRNA) levels of Pparg2 in a dose-dependent manner (Figure 1A). GGOH also increased the protein levels of not only PPAR $\gamma 2$, but also PPAR $\gamma 1$ in C3H10T1/2 cells (Figure 1B). It was also confirmed that GGOH induced Pparg 2 expression in 3T3L1 cells (Figure 1C).

The effect of GGOH on the cellular response to the PPAR $\gamma$ agonist, rosiglitazone, was then examined. Rosiglitazone induces the expression of a cascade of adipogenic transcription factors leading to lipid accumulalion and adipogenic differentiation (18). Here, GGOH synergistically enhanced the 

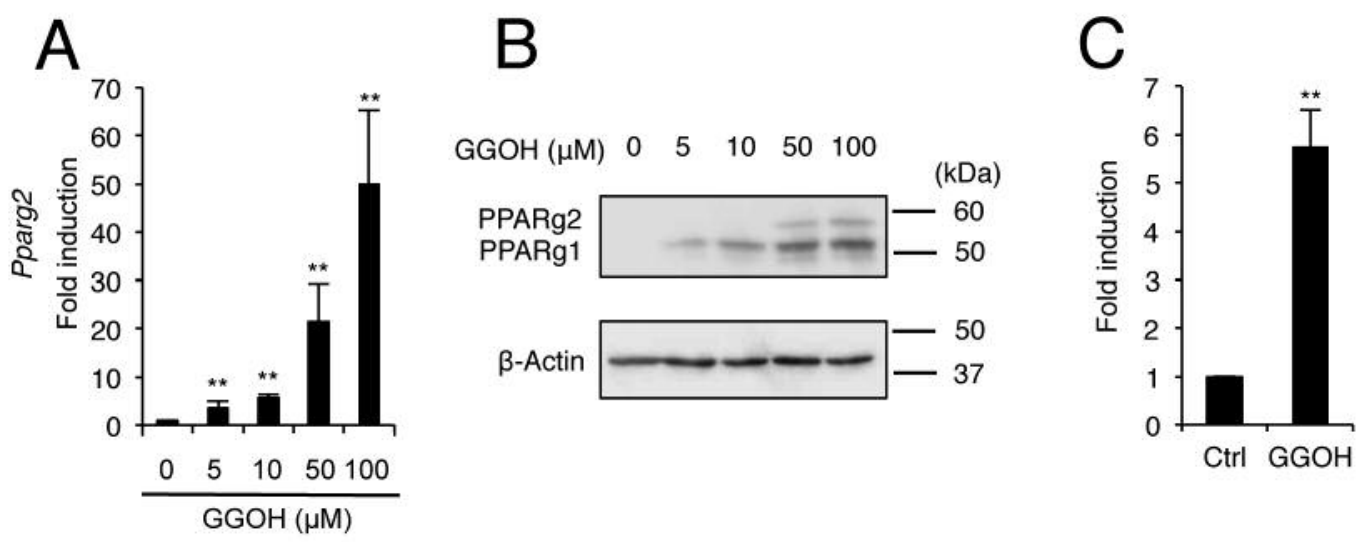

Figure 1. Geranylgeraniol (GGOH) induces peroxisome proliferator-activated receptor $\gamma(P P A R \gamma)$ expression. C3H10T1/2 cells were treated with 0, 5, 10, 50, or $100 \mu M$ GGOH after which the messenger RNA (mRNA) level of Pparg2 (A) was determined by quantitative real-time PCR and protein levels of PPAR 1 and PPAR 2 (B) were assessed by western blot analysis on day 2. GGOH treatment $(50 \mu M)$ increased the mRNA level of Pparg2 in 3T3-L1 cells on day $2(C)$. The data are expressed as the mean $\pm S D(n=3)$. **Significantly different at $p<0.01$ versus $0 \mu M$ GGOH or control (Ctrl; DMSO) treatment.

induction of Pparg2 by rosiglitazone in C3H10T1/2 (Figure 2A) and 3T3-L1 cells (Figure 2E). GGOH also enhanced the expression of classical adipogenic marker genes, Fabp4, Cebpa, and Adipoq, following treatment with rosiglitazone (Figure 2B, C, and F-H). GGOH treatment led to an increase in the number and size of lipid droplets induced by the PPAR $\gamma$ agonist. However, GGOH failed to increase the transcriptional activity of Pparg 2 assessed by a luciferase reporter assay (Figure 2J), suggesting that GGOH does not directly affect Pparg2 transcriptional activity, at least on the FABP4 promoter.

GGOH prevents reduction of Pparg2 expression by statin. Finally, it was examined whether GGOH rescues the suppressive effect of statin on PPAR $\gamma$ expression. In the absence of GGOH, simvastatin dose-dependently inhibited rosiglitazone-induced Pparg2 expression. However, in the presence of $\mathrm{GGOH}$, simvastatin failed to inhibit Pparg2 expression (Figure 3A).

\section{Discussion}

The rapidly rising prevalence of diabetes in middle- and lowincome countries has resulted in a more urgent need for inexpensive and effective treatments for diabetes (1). GGOH is a safe, inexpensive, natural, and orally-ingestible compound. In the present study, we showed that GGOH increased the expression of PPAR $\gamma$ (Figure 1). Therefore, GGOH, especially when combined with a PPAR $\gamma$ agonist may be a potential drug for the prevention or treatment of diabetes. Needless to say, further experiments are needed to determine the downstream targets of GGOH, as well as to assess the effect of GGOH in an in vivo diabetes model.

A broad range of synthetic PPAR $\gamma$ ligands have been developed. The most commonly used synthetic PPAR $\gamma$ agonists belong to the thiazolidinedione class of anti-diabetic medicines. The synthetic ligands troglitazone, rosiglitazone, and pioglitazone have already been applied clinically for the treatment of type 2 DM. These therapeutics make use of the ability of synthetic ligands to increase insulin sensitivity and to lower blood sugar levels (6). However, troglitazone was recently withdrawn from the market due to severe adverse side-effects in the liver (7). Since here GGOH dramatically enhanced the effects of a PPAR $\gamma$ ligand (Figure 2), GGOH may reduce the effective dosage and side-effects of these synthetic agents.

Several naturally-derived compounds including emodin (21), phloretin (22), and ginsenosides $(23,24)$ have been reported to increase adipogenesis and elevate the expression of adipocytokines capable of stimulating insulin sensitivity. More specifically, ginsenoside 20(S)-protopanaxatriol and emodin were demonstrated to be ligands for PPAR $\gamma(21,23)$. Altogether this suggests that it may be possible to carefully manage dietary intake to enrich for foods that are high in GGOH and thus harness natural PPAR $\gamma$ ligand effects in diabetes prevention.

Statins are used as a frontline therapy to lower plasma cholesterol and prevent cardiovascular disease. Many clinical studies have demonstrated that statins are very effective in reducing death and disorders caused by cardiovascular disease (25-29). However, recent data show that long-term statin therapy is associated with an increased occurrence and risk of 


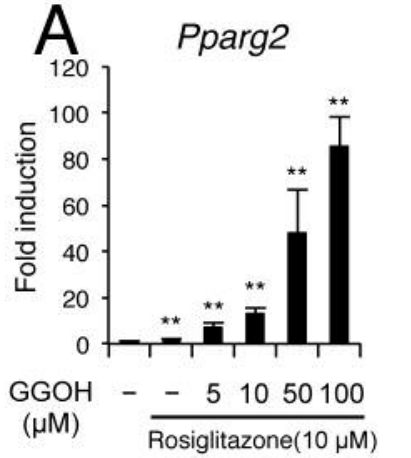

D

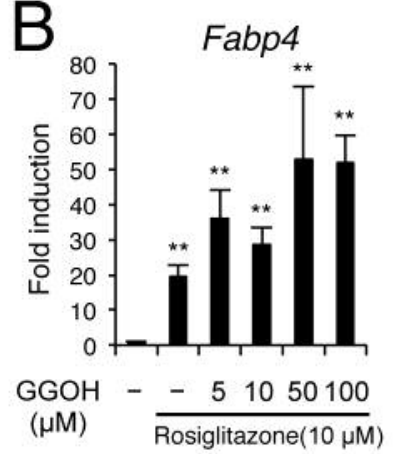

C

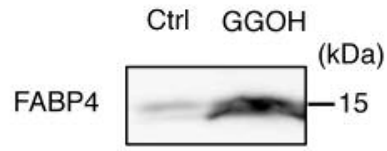

$\beta$-Actin

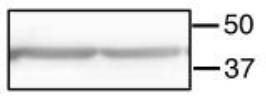

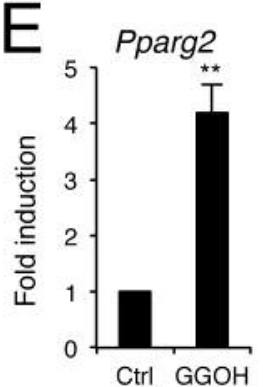

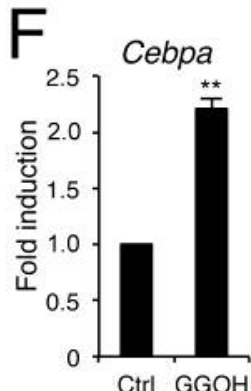

$\mathrm{GGOH}(\mu \mathrm{M}) 0$

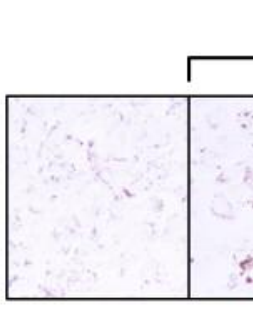

Rosiglitazone $(10 \mu \mathrm{M})$
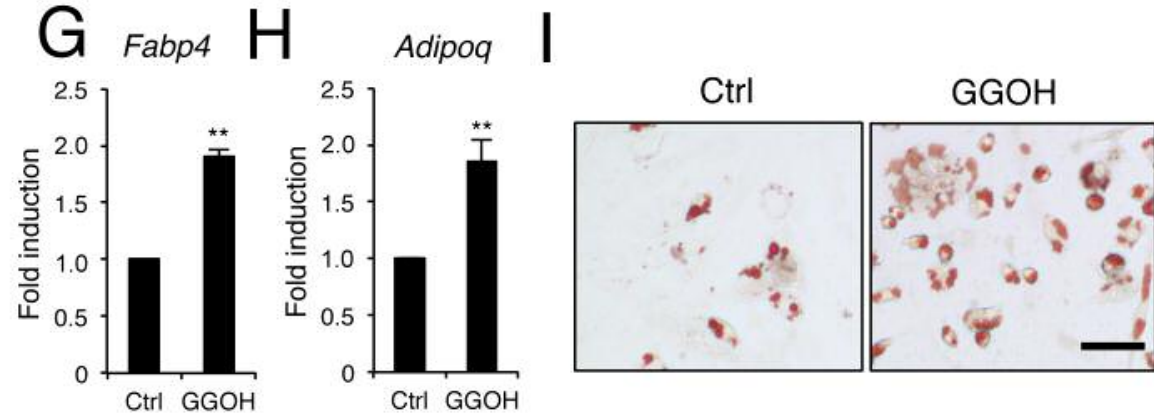

$J$

FABP4-Luc activity

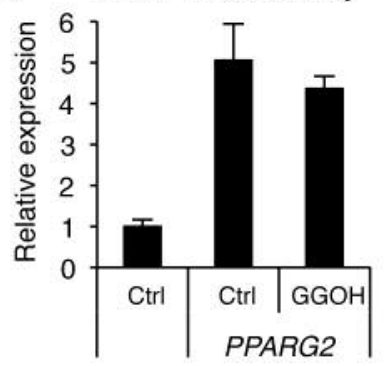

Figure 2. Geranylgeraniol (GGOH) enhances adipocyte differentiation of C3H10T1/2 mouse embryonic fibroblasts and 3T3-L1 pre-adipocytes. C3H10T1/2 cells were treated with 0, 5, 10, 50, or $100 \mu \mathrm{M} G G O H$ with or without $10 \mu M$ rosiglitazone. The mRNA levels of A: Peroxisome proliferator-activated receptor $\gamma 2$ (Pparg2) and B: Fatty acid binding protein 4 (Fabp4) were determined on day 2. C: GGOH treatment (50 $\mu M$ ) enhanced the protein expression of FABP4 induced by $10 \mu \mathrm{M}$ rosiglitazone in C3H10T1/2 cells after 3 days. D: Cells were treated with 0, 5, 10, 50 , or $100 \mu \mathrm{M} \mathrm{GGOH}$ with or without $10 \mu \mathrm{M}$ rosiglitazone. Adipocytes were stained with oil red $O$ on day 7. 3T3-L1 cells were treated with $50 \mu M$ GGOH with or without $10 \mu \mathrm{M}$ rosiglitazone and the mRNA levels of E: Pparg2, F: CCAAT-enhancer-binding protein $\alpha$ (Cebpa), G: Fabp4 and H: adiponectin, C1Q and collagen domain-containing (Adipoq) were determined after 2 days. I: Adipocytes were stained with oil red $O$ on day 7. J: C3H10T1/2 cells were transfected with PPARG2 or an empty vector along with FABP4-luciferase vector and treated with or without $50 \mu M$ $G G O H$. Luciferase activity was determined on day 1. The data are expressed as the mean $\pm S D(n=3)$. **Significantly different at $p<0.01$ versus $0 \mu \mathrm{M}$ GGOH or control (Ctrl; DMSO) treatment. Scale bar indicates $20 \mu \mathrm{m}$ (D and I).

insulin resistance and type 2 DM (30-33). Since 2012, the United States Food and Drug Administration requires statin drug package inserts to include a warning of the risk of type 2 DM (34). Reduction of GGPP-induced PPAR $\gamma$ expression by inhibition of the isoprenoid biosynthetic pathway may be involved in the occurrence of insulin resistance and type 2 DM. Since GGOH counteracts the effect of statin on PPAR $\gamma$ (Figure 3), this also suggests that GGOH, a GGPP precursor, may be a potential drug for the prevention or treatment of statin-induced diabetes without interfering with the beneficial plasma cholesterol-lowering effects of statin.

\section{Conflicts of Interest}

The Authors declare that they have no conflict of interests in regard to this study. 


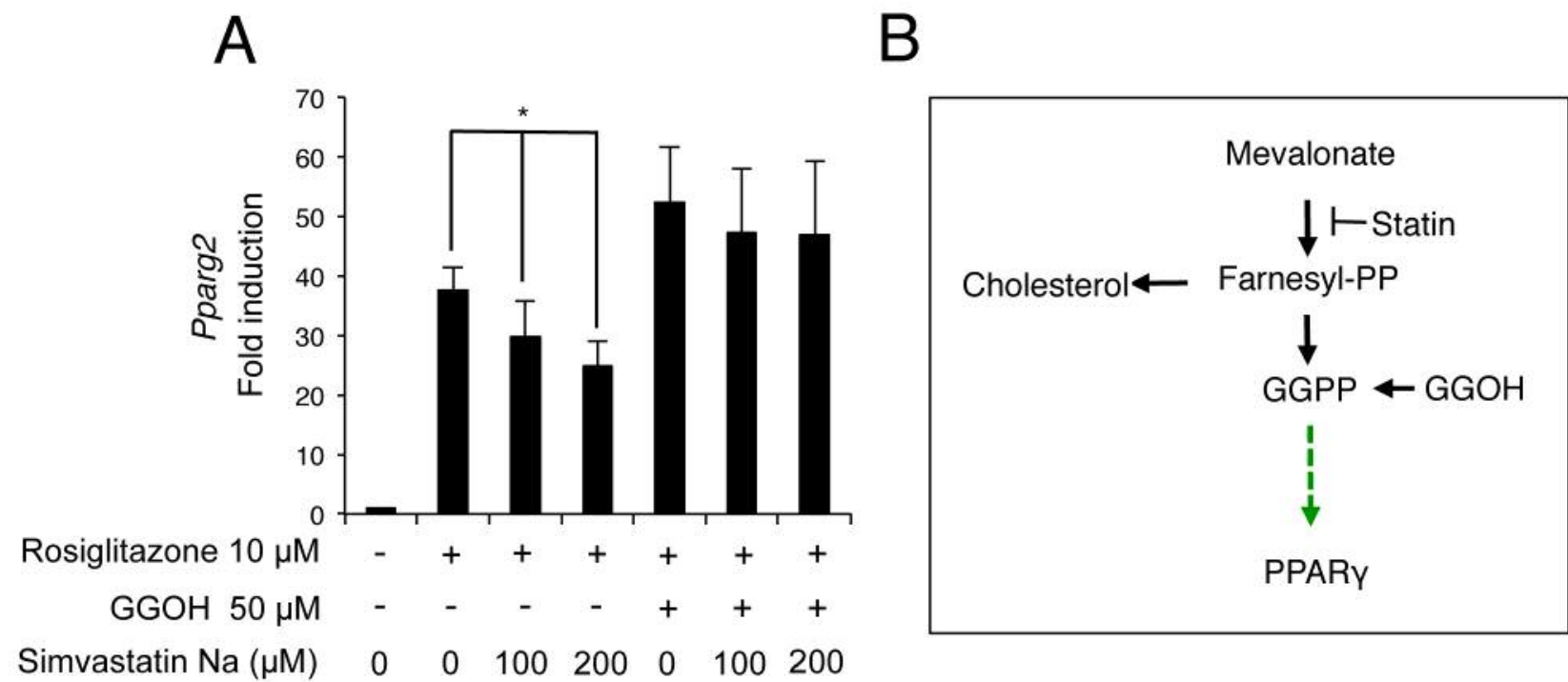

Figure 3. Geranylgeraniol (GGOH) rescues the inhibition of Peroxisome proliferator-activated receptor $\gamma(P P A R \gamma)$ by simvastatin. A: C3H10T1/2 cells were treated with rosiglitazone, GGOH and simvastatin, alone and in combination for 3 days. The mRNA level of Pparg 2 was determined by quantitative real-time polymerase chain reaction. The data are expressed as the mean $\pm S D(n=3)$. *Significantly different at $p<0.05$. B: Model for GGOH regulation of PPAR $\gamma$ expression.

\section{Acknowledgements}

This study was supported by the Suzuken Memorial Foundation (S. K.).

\section{References}

1 World Health Organisation: Global Report on Diabetes 2016 WHO. Geneva, 2016.

2 Fajas L, Auboeuf D, Raspe E, Schoonjans K, Lefebvre AM, Saladin R, Najib J, Laville M, Fruchart JC, Deeb S, Vidal-Puig A, Flier J, Briggs MR, Staels B, Vidal H and Auwerx J: The organization, promoter analysis, and expression of the human PPARgamma gene. J Biol Chem 272: 18779-18789, 1997.

3 Elbrecht A, Chen Y, Cullinan CA, Hayes N, Leibowitz M, Moller DE and Berger J: Molecular cloning, expression and characterization of human peroxisome proliferator activated receptors gamma 1 and gamma 2. Biochem Biophys Res Commun 224: 431-437, 1996.

4 Lemberger $\mathrm{T}$, Desvergne $\mathrm{B}$ and Wahli W: Peroxisome proliferator-activated receptors: A nuclear receptor signaling pathway in lipid physiology. Annu Rev Cell Dev Biol 12: 335363,1996

5 Grommes C, Landreth GE and Heneka MT: Antineoplastic effects of peroxisome proliferator-activated receptor gamma agonists. Lancet Oncol 5: 419-429, 2004.

6 Berger J, Bailey P, Biswas C, Cullinan CA, Doebber TW, Hayes NS, Saperstein R, Smith RG and Leibowitz MD: Thiazolidinediones produce a conformational change in peroxisomal proliferator-activated receptor-gamma: binding and activation correlate with antidiabetic actions in $\mathrm{db} / \mathrm{db}$ mice. Endocrinology 137: 4189-4195, 1996.
7 Willson TM, Brown PJ, Sternbach DD, and Henke BR: The PPARs: From orphan receptors to drug discovery. J Med Chem 43: 527-550, 2000

8 Muraguchi T, Okamoto K, Mitake M, Ogawa H and Shidoji Y: Polished rice as natural sources of cancer-preventing geranylgeranoic acid. J Clin Biochem Nutr 49: 8-15, 2011.

9 Frenkel J, Rijkers GT, Mandey SH, Buurman SW, Houten SM, Wanders RJ, Waterham HR and Kuis W: Lack of isoprenoid products raises ex vivo interleukin-1beta secretion in hyperimmunoglobulinemia $\mathrm{D}$ and periodic fever syndrome. Arthritis Rheum 46: 2794-2803, 2002.

10 Crick DC, Andres DA and Waechter CJ: Farnesol is utilized for protein isoprenylation and the biosynthesis of cholesterol in mammalian cells. Biochem Biophys Res Commun 211: 590-599, 1995.

11 Walker K and Olson MF: Targeting Ras and Rho GTPases as opportunities for cancer therapeutics. Curr Opin Genet Dev 15: 62-68, 2005.

12 Weivoda MM and Hohl RJ: Geranylgeranyl pyrophosphate stimulates PPARgamma expression and adipogenesis through the inhibition of osteoblast differentiation. Bone 50: 467-476, 2012.

13 Endo A, Tsujita Y, Kuroda M and Tanzawa K: Inhibition of cholesterol synthesis in vitro and in vivo by ML-236A and ML236B, competitive inhibitors of 3-hydroxy-3-methylglutarylcoenzyme A reductase. Eur J Biochem 77: 31-36, 1977.

14 Baba TT, Ohara-Nemoto Y, Miyazaki T and Nemoto TK: Involvement of geranylgeranylation of Rho and Rac GTPases in adipogenic and RANKL expression, which was inhibited by simvastatin. Cell Biochem Funct 31: 652-659, 2013.

15 Kokabu S, Nakatomi C, Matsubara T, Ono Y, Addison WN, Lowery JW, Urata M, Hudnall AM, Hitomi S, Nakatomi M, Sato 
T, Osawa K, Yoda T, Rosen V and Jimi E: The transcriptional co-repressor TLE3 regulates myogenic differentiation by repressing the activity of the MyoD transcription factor. J Biol Chem 292: 12885-12894, 2017.

16 Kokabu S, Sato T, Ohte S, Enoki Y, Okubo M, Hayashi N, Nojima J, Tsukamoto S, Fukushima Y, Sakata Y, Katagiri T, Rosen $\mathrm{V}$ and Yoda T: Expression of TLE3 by bone marrow stromal cells is regulated by canonical Wnt signaling. FEBS Lett 588: 614-619, 2014.

17 Kokabu S, Gamer L, Cox K, Lowery J, Tsuji K, Raz R, Economides A, Katagiri T and Rosen V: BMP3 suppresses osteoblast differentiation of bone marrow stromal cells via interaction with ACVR2B. Mol Endocrinol 26: 87-94, 2012.

18 Kokabu S, Nguyen T, Ohte S, Sato T, Katagiri T, Yoda T and Rosen V: TLE3, transducing-like enhancer of split 3, suppresses osteoblast differentiation of bone marrow stromal cells. Biochem Biophys Res Commun 438: 205-210, 2013.

19 Kokabu S, Nojima J, Kanomata K, Ohte S, Yoda T, Fukuda T and Katagiri T: Protein phosphatase magnesium-dependent 1Amediated inhibition of BMP signaling is independent of SMAD dephosphorylation. J Bone Miner Res 25: 653-660, 2010.

20 Kasturi R and Wakil SJ: Increased synthesis and accumulation of phospholipids during differentiation of 3T3-L1 cells into adipocytes. J Biol Chem 258: 3559-3564, 1983.

21 Yang Y, Shang W, Zhou L, Jiang B, Jin H and Chen M: Emodin with PPARgamma ligand-binding activity promotes adipocyte differentiation and increases glucose uptake in 3T3-Ll cells. Biochem Biophys Res Commun 353: 225-230, 2007.

22 Hassan M, El Yazidi C, Landrier JF, Lairon D, Margotat A and Amiot MJ: Phloretin enhances adipocyte differentiation and adiponectin expression in 3T3-L1 cells. Biochem Biophys Res Commun 361: 208-213, 2007.

23 Han KL, Jung MH, Sohn JH and Hwang JK: Ginsenoside 20Sprotopanaxatriol (PPT) activates peroxisome proliferatoractivated receptor gamma (PPARgamma) in 3T3-L1 adipocytes. Biol Pharm Bull 29: 110-113, 2006.

24 Shang W, Yang Y, Jiang B, Jin H, Zhou L, Liu S and Chen M: Ginsenoside Rb1 promotes adipogenesis in 3T3-L1 cells by enhancing PPARgamma2 and C/EBPalpha gene expression. Life Sci 80: 618-625, 2007.

25 Kain V, Kapadia B, Misra P and Saxena U: Simvastatin may induce insulin resistance through a novel fatty acid mediated cholesterol independent mechanism. Sci Rep 5: 13823, 2015.
26 Grundy SM and Vega GL: Influence of mevinolin on metabolism of low density lipoproteins in primary moderate hypercholesterolemia. J Lipid Res 26: 1464-1475, 1985.

27 Hoeg JM, Maher MB, Zech LA, Bailey KR, Gregg RE, Lackner KJ, Fojo SS, Anchors MA, Bojanovski M, Sprecher DL and Brewer HB Jr.: Effectiveness of mevinolin on plasma lipoprotein concentrations in type II hyperlipoproteinemia. Am J Cardiol 57: 933-939, 1986.

28 Illingworth DR and Sexton GJ: Hypocholesterolemic effects of mevinolin in patients with heterozygous familial hypercholesterolemia. J Clin Invest 74: 1972-1978, 1984.

29 Tikkanen MJ and Nikkila EA: Current pharmacologic treatment of elevated serum cholesterol. Circulation 76: 529-533, 1987.

30 Sattar N, Preiss D, Murray HM, Welsh P, Buckley BM, de Craen AJ, Seshasai SR, McMurray JJ, Freeman DJ, Jukema JW, Macfarlane PW, Packard CJ, Stott DJ, Westendorp RG, Shepherd J, Davis BR, Pressel SL, Marchioli R, Marfisi RM, Maggioni AP, Tavazzi L, Tognoni G, Kjekshus J, Pedersen TR, Cook TJ, Gotto AM, Clearfield MB, Downs JR, Nakamura H, Ohashi Y, Mizuno K, Ray KK and Ford I: Statins and risk of incident diabetes: a collaborative meta-analysis of randomised statin trials. Lancet 375: 735-742, 2010.

31 Corrao G, Ibrahim B, Nicotra F, Soranna D, Merlino L, Catapano AL, Tragni E, Casula M, Grassi G and Mancia G: Statins and the risk of diabetes: evidence from a large population-based Cohort study. Diabetes Care 37: 2225-2232, 2014.

32 Macedo AF, Douglas I, Smeeth L, Forbes H and Ebrahim S: Statins and the risk of type 2 diabetes mellitus: Cohort study using the UK clinical practice pesearch datalink. BMC Cardiovasc Disord 14: 85, 2014.

33 Bell DS, DiNicolantonio JJ and O'Keefe JH: Is statin-induced diabetes clinically relevant? A comprehensive review of the literature. Diabetes Obes Metab 16: 689-694, 2014.

34 Brault M, Ray J, Gomez YH, Mantzoros CS and Daskalopoulou SS: Statin treatment and new-onset diabetes: a review of proposed mechanisms. Metabolism 63: 735-745, 2014. 